\title{
Comprehensive Toxic Plants-Phytotoxins Database and Its Application in Assessing Aquatic Micropollution Potential
}

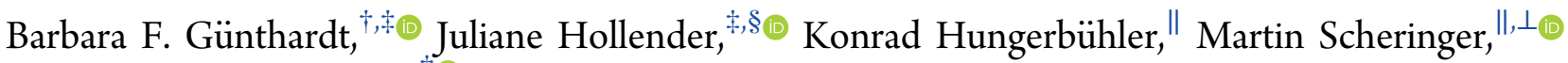 \\ and Thomas D. Bucheli*, ${ }^{\dagger}$ (i) \\ ${ }^{\dagger}$ Environmental Analytics, Agroscope, Reckenholzstrasse 191, 8046 Zürich, Switzerland \\ ${ }^{\ddagger}$ Institute of Biogeochemistry and Pollutant Dynamics, ETH Zurich, Universitätsstrasse 16, 8092 Zürich, Switzerland \\ ${ }^{\S}$ Swiss Federal Institute of Aquatic Science and Technology (Eawag), Überlandstrasse 133, 8600 Dübendorf, Switzerland \\ "Institute for Chemical and Bioengineering, ETH Zurich, Wolfgang-Pauli-Strasse 10, 8093 Zürich, Switzerland \\ ${ }^{\perp}$ RECETOX, Masaryk University, Kamenice 753/5, 62500 Brno, Czech Republic
}

Supporting Information

\begin{abstract}
The production of toxic plant secondary metabolites (phytotoxins) for defense is a widespread phenomenon in the plant kingdom and is even present in agricultural crops. These phytotoxins may have similar characteristics to anthropogenic micropollutants in terms of persistence and toxicity. However, they are only rarely included in environmental risk assessments, partly because a systematic overview of phytotoxins is missing. Here, we present a newly developed, freely available database, Toxic Plants-PhytoToxins (TPPT), containing 1586 phytotoxins of potential ecotoxicological relevance in Central Europe linked to 844 plant species. Our database summarizes phytotoxin patterns in plant species and provides detailed biological and chemical information as well as in silico estimated properties. Using the database, we evaluated phytotoxins regarding occurrence, approximated from the frequencies of Swiss plant species; environmental behavior based on aquatic persistence and mobility; and toxicity. The assessment showed that over $34 \%$ of all phytotoxins are potential aquatic micropollutants and should be included in environmental investigations.
\end{abstract}

KEYWORDS: natural toxins, poisonous plants, invasive species, aquatic pollution, risk assessment

\section{INTRODUCTION}

Today, many anthropogenic chemicals are recognized as environmentally relevant micropollutants because of their persistence and toxicity. Of special concern are anthropogenic chemicals that are directly applied to the environment (e.g., pesticides $)^{1}$ or released through wastewater treatment plants (WWTP, e.g., pharmaceuticals and the ingredients of personalcare products). ${ }^{2,3}$ Consequently, monitoring programs have emerged to systematically investigate the appearance of these chemicals. Among all released chemicals, persistent, bioaccumulative, and toxic (PBT) substances are of particular importance because they can concentrate in the food chain and cause long-term effects. ${ }^{4,5}$ Recently, persistent, mobile, and toxic (PMT) substances have also increasingly come into focus as a result of the threat they pose to water quality. ${ }^{6,7}$ These relatively polar PMT substances are not bioaccumulative and were often neglected in traditional screenings until concerns arose related to them being much less effectively removed in WWTPs and thus being highly problematic for the aquatic environment. $^{8}$

In contrast to anthropogenic PBT and PMT compounds, plant-produced compounds with similar properties have received little attention as potential micropollutants, although the production of toxic plant secondary metabolites (PSMs) is common in the plant kingdom and even takes place in agricultural crops. These so-called phytotoxins, including allelochemicals, allergens, hallucinogens, fatal toxins, and biopesticides, constitute a category of natural compounds with various toxic effects and diverse molecular structures (e.g., alkaloids, terpenes, phenylpropanoids, and polyketides). ${ }^{9,10}$ Phytotoxins might contribute to mixture toxicities and locally even outcompete anthropogenic chemicals in their overall risk because of constant production and comparatively high concentrations in plants. ${ }^{11}$ Particularly, plants with high local abundance often induced by human activity, such as crops, invasive neophytes, or foreign ornamental garden plants, might be of concern.

The environmental behavior of phytotoxins has been investigated only for a limited number of compounds. Indeed, case studies on single phytotoxins have demonstrated relevant exposure, for instance in the case of strongly toxic glycoalkaloids produced from potato (Solanum tuberosum), ${ }^{12}$ estrogenic isoflavones from red clover grasslands (Trifolium pratense), ${ }^{13}$ or the carcinogenic ptaquiloside from the nonagricultural bracken fern (Pteridium aquilinum). ${ }^{14}$

Environmental investigations including a larger number of phytotoxins are often hindered by the high number of structurally diverse phytotoxins and a lack of analytical standards and validated methodologies. ${ }^{11,15}$ Another prerequisite for a systematic investigation of a wide range of phytotoxins is the availability of a comprehensive database

Received: March 29, 2018

Revised: June 8, 2018

Accepted: June 26, 2018

Published: June 26, 2018 
including both plants and their toxins. The importance of phytotoxins and PSMs in general in specific domains such as pest management, the culinary industry, the perfume industry, and medicinal research has led to the development of several excellent databases. Because they are often optimized for the specific needs of certain communities (e.g., SuperToxic ${ }^{16}$ and Super Natural $\mathrm{II}^{17}$ in medicinal research), they are of limited use in other fields. For environmental chemists and regulators, the Aggregated Computational Toxicology Online Resource $(\mathrm{ACToR})^{18}$ from the U.S. Environmental Protection Agency (EPA) is a specialized chemical database, but like most of them, it does not provide connections between PSMs and plant species. In this respect, the KNApSAcK database ${ }^{19}$ is useful because it contains chemical information and especially plant-species-metabolite relationships. Databases on toxic plants can also be useful, such as the compendium from the European Food Safety Authority (EFSA $)^{20}$ or the Clinical Toxicology (CliniTox) database, but they generally contain limited chemical information. ${ }^{21}$ Indeed, textbooks ${ }^{22-25}$ are often the most detailed data sources regarding the combination of plant species, phytotoxins, and toxicity information, but printed information is of limited use for efficient and systematic computer-based data analysis. Overall, an environmental risk assessment remains challenging (if not impossible) because of the lack of databases containing phytotoxins, plant species, toxicities, and environmentally relevant chemical properties.

Here, we describe the compilation of potentially relevant toxic plants in Central Europe together with their corresponding phytotoxins in a newly developed and freely available database: Toxic Plants-PhytoToxins (TPPT, available for download from https://www.agroscope.admin.ch/agroscope/ en/home/publications/apps/tppt.html). The established database contains 844 plant species with biological information, including, among others, plant names, distribution, human toxicity, and vegetation type, and 1586 phytotoxins with chemical information covering compound identification, structural characterization, in silico estimated physicochemical and toxicological properties, and corresponding references. To the best of our knowledge, the TPPT database is unique in summarizing phytotoxin patterns in individual plant species while at the same time providing detailed information about both plants and toxins. The TPPT database was then used for a preliminary aquatic risk assessment, in which the phytotoxins were evaluated on the basis of (1) their occurrence, approximated from the plant species' frequencies in Switzerland, (2) their environmental behavior, including aquatic persistence and mobility according to Arp et al., ${ }^{6}$ and (3) their acute rodent and aquatic toxicity. The applied prioritization procedure is a first important step to differentiating phytotoxins on the basis of their properties and identifying critical PSM classes that should be included in surface-water screenings.

\section{MATERIALS AND METHODS}

TPPT-Database Purpose and Range Definition. The TPPT database was designed to facilitate environmental risk assessments of phytotoxins, such as their prioritization according to their relevance for the aquatic environment, and therefore covers plant species and phytotoxins together with exposure- and effect-related information. Although we acknowledged the existence of several good databases, it was not feasible to start from an existing database, and we preferred to start ab initio. In the first step, all higher plant species that (i) occur in Switzerland as naturally growing plants, invasive neophytes, agricultural plants, or ornamental garden plants and (ii) have possible negative effects due to their produced PSMs affecting humans, animals (including husbandry animals), the aquatic ecosystem, or other plants (allelopathy) were included in the TPPT database. Agricultural plants that do not grow in Switzerland but still have global importance were also included, such as the cacao tree, cotton, and the most important coffee plant (Coffea arabica). ${ }^{23}$ The vegetation of Switzerland was chosen because it covers several altitudinal zones from foothill to alpine, is species-rich (over 3600 different higher plant species), and is largely representative for Central Europe. ${ }^{26-28}$ The southern part of the country is further influenced by the Mediterranean climate. We focus on higher plant species, and therefore, other natural toxin sources, such as algae or cyanobacteria, are not covered by the TPPT database, although they also produce relevant natural toxins. ${ }^{29,30}$ In a second step, the major toxic PSMs present in the previously selected plants were identified, added to the database, and if the data were available, complemented with minor toxic PSMs. The emphasis was placed on PSMs with known adverse effects. Although primary metabolites could also cause toxicity, in contrast to PSMs they do not act as defense compounds but serve in essential plant physiological functions, notably photosynthesis and carbon, nitrogen, and fatty acid metabolism. ${ }^{9,31}$ This second step was often vague, because plants mostly produce several different PSM classes composed of high numbers of compounds that mostly have not yet fully been resolved and identified and of which several might be toxic. Moreover, the phytotoxin-plant-species relationship regularly varies between different regions, plant chemotypes, and seasons. Aside from this natural complexity, the term "toxin" is not clearly defined, because toxicity depends on dosage, and there are other forms of negative effects apart from acute toxicity, such as allergenic or mutagenic activity. Clearly, many PSMs also have beneficial effects on other organisms or humans or are not phytotoxic at all. ${ }^{23,32}$ Finally, the research knowledge on toxic substances is frequently limited, and the available literature is partially inconsistent. The degree of toxicity and the plant abundance are two major factors that lead to research into a plant species, and often only a few plant species are investigated within a genus. As a consequence of these uncertainties, no concentrations, either in the plant species or in the environment, are included in the TPPT database, and PSMs with uncertain occurrence, either due to missing data or due to extreme natural variations, are marked accordingly.

Data Sources. Table 1 summarizes data sources that were combined from various scientific fields to compile first the toxic plant species together with their biological information, followed by the corresponding phytotoxin-toxic-plant-species relationships and phytotoxin structures. Besides these main data sources, a literature search was conducted to find journal articles specific for a plant species or PSM class and complement the phytotoxin-plant-species relationships. The data compilation is further detailed in the Supporting Information (SI), and individual references for each phytotoxin are provided in the databases. For the chemical information, we used PubChem $^{33}$ and ChemSpider, ${ }^{34}$ and the molecular structures, including, if available, the stereochemistry, were compared between the two databases to avoid errors in the structure identification. The identifiers of these two chemical databases were also included, because they contain complementary information, as well as references to other databases. In cases where the possible structures or their stereochemistry were different, the structure from PubChem was preferred. Experimentally determined toxicity end points were also collected (for details, see the SI), resulting in a total of 318 measurements for 184 phytotoxins. All information was manually assembled from the mentioned sources. In a final step, all data fields were quality-controlled regarding the plausibility of the content and possible spelling mistakes.

TPPT-Database Setup. The setup of the TPPT database is visualized in Figure 1. The TPPT database is structured around two main data tables, the toxic-plant-species table and the phytotoxin table, which are indexed by internal numbers, and a superior table that relates the phytotoxins to the plant species. The toxic-plant table contains 17 data fields with biological information covering plant- 
Table 1. Data Sources Used to Compile the Information in the Toxic-Plants-Phytotoxins (TPPT) Database ${ }^{a}$

information
plant species and biological in-
formation (all naturally growin
plant species in Switzerland)
plant species and biological in-
formation (plant species toxic
to humans)
plant species and biological in-
formation (plant species toxic
to humans)
plant species (invasive plant
species)

phytotoxin-toxic-plant-species relationships

\author{
data sources \\ compendium from Lauber et al. ${ }^{43}$ \\ textbook from Teuscher and Lindequist ${ }^{23}$ \\ textbook from Roth et al. ${ }^{24}$
}

Clinical Toxicology (CliniTox) database ${ }^{21}$

National Data and Information Center on the Swiss Flora (info flora) ${ }^{28}$

Swiss Ordinance on the Handling of Organisms in the Environment (Annex 2, list with prohibited invasive alien organisms $)^{49}$

EU Regulation No. 1143/2014 (list of invasive alien species of Union concern $)^{50}$ textbook from Teuscher and Lindequist ${ }^{23}$

textbook from Roth et al. ${ }^{24}$

KNApSAcK database ${ }^{51}$

compendium from the European Food Safety Authority (EFSA) ${ }^{20}$

CliniTox database ${ }^{21}$

Web of Science literature search (individual scientific journals)

${ }^{a}$ For data-compilation details, see the SI.

species name and systematics as well as plant distribution, habitat, toxicity, and categorization (i.e., ornamental garden plant, agricultural plant, invasive neophyte, or naturally growing plant). The phytotoxin table contains 14 data fields for phytotoxin identification and chemical information and also the literature references for the phytotoxintoxic-plant-species relationships. The phytotoxin-toxic-plant-speciesrelationships table defines the phytotoxin patterns, including the composition data field, which gives more information on the major toxin, uncertainty, and natural variations, as far as they are known (see Table 2). Additionally, a remarks table contains extra notes in a comment field and an approximate score describing the available scientific knowledge for a given plant genus. Table 2 gives a detailed content description and illustrative examples for each data field in the main database tables. Additional information, including experimental toxicity data, in silico property estimations, aquatic micropollution potential analysis, and corresponding literature references, are organized in subtables in the phytotoxin table.
The TPPT database was built with the common and fast opensource database software SQLite. ${ }^{35}$ This SQL-based software supports most of standard SQL and, together with the file-based setup, allows individual adaptation of the TPPT database for a given purpose, the creation of different searches, or the exporting of single parts of the TPPT database. SQLite can be used with different interfaces, some of which are also free, and the database can also be accessed from different programming languages, such as $\mathrm{R}^{36,37}$ Additionally, we converted the database into the simpler, user-friendly Excel spreadsheet. The TPPT database can be downloaded from the following homepage: https://www.agroscope.admin.ch/agroscope/ en/home/publications/apps/tppt.html.

In Silico Estimations of Physicochemical Properties and Toxicities. In environmental risk assessments, several properties of a substance are needed to estimate its exposure and effects. Because experimentally measured data are often not available for phytotoxins, we included outputs from four different estimations tools: The often applied software Estimation Program Interface (EPI) Suite from the U.S. EPA was used to predict most physicochemical properties, including several distribution and degradation parameters. ${ }^{38}$ Although EPI Suite is often criticized for being inaccurate, it also has major advantages: it requires only the SMILES structure as input, it can be run in batch mode, and it is freely available. ${ }^{6,39}$ Additional physicochemical parameters not available through EPI Suite, in particular the $\mathrm{p} K_{x}$, were predicted with $\mathrm{ACD} /$ Percepta, ACD/Lab's Percepta Predictor software (Advanced Chemistry Development Inc.). ${ }^{40}$ To characterize mammalian toxicity, ProTox was used, which predicts the rodent oral toxicity as the median lethal dose $\left(\mathrm{LD}_{50}\right)$ in milligrams per kilogram of body weight. ${ }^{41}$ The ecotoxicity was predicted using the U.S. EPA's ecological structure-activity relationships (ECOSAR) predictive model (version 2.0), which estimates acute and chronic toxicity at the lethal median concentrations $\left(\mathrm{LC}_{50}\right)$ and chronic values for three model organisms: fish, daphnia, and green algae. ${ }^{42}$ For all these tools, the applicability is limited, covering mostly only organic, uncharged, low-molecular weight (often $<1000 \mathrm{~g} / \mathrm{mol}$ ) substances, and thus do not apply for all phytotoxins. Consequently, an applicability specification is given for each phytotoxin, but it should be noted that all predictions are fraught with some uncertainty. However, they fulfill the purpose of primary prioritization because there are also other uncertainties (e.g. those related to plants, see above). More details on the prediction tools, the included information, and descriptions of the data fields are provided in the SI.

Aquatic Micropollution Potential Assessment. We developed a prioritization system to evaluate the phytotoxins on the basis of their aquatic micropollution potentials, including (i) the phytotoxin occurrence, (ii) the environmental behavior based on aquatic

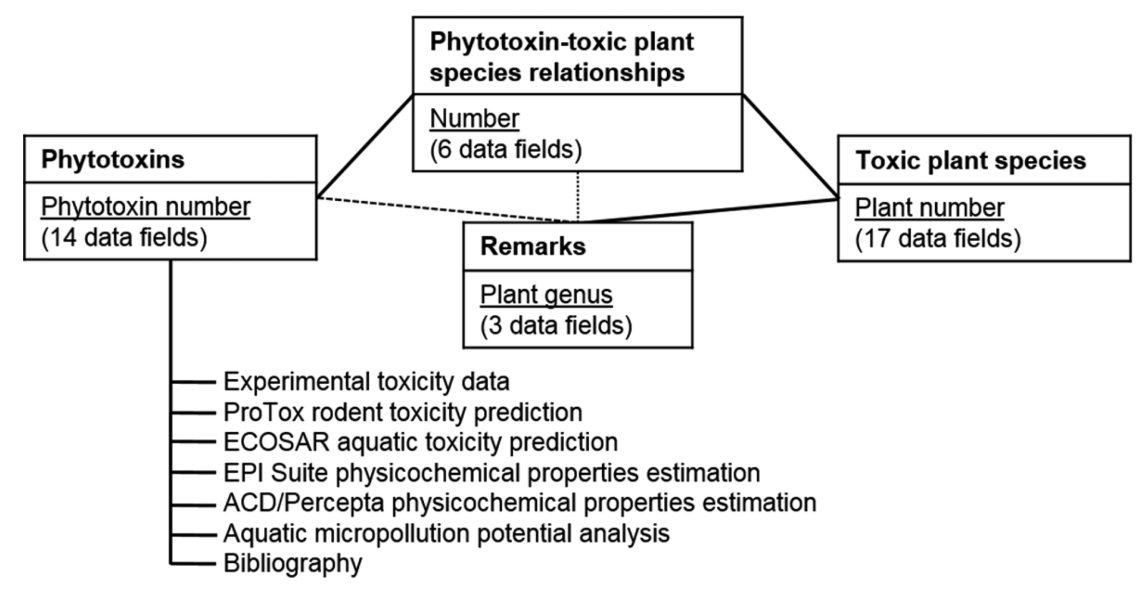

Figure 1. Visualization of the toxic-plant-phytotoxin (ТРPT)-database setup with all the included tables. The four main tables are given together with the underlined primary keys and the numbers of included data fields in brackets. Individual fields are described in Table 2. Tables with additional information (i.e., experimental toxicity data, in silico property estimations, aquatic micropollution potential analysis, and bibliography) are subtables in the phytotoxin table and are described further in Tables S1-S6. 


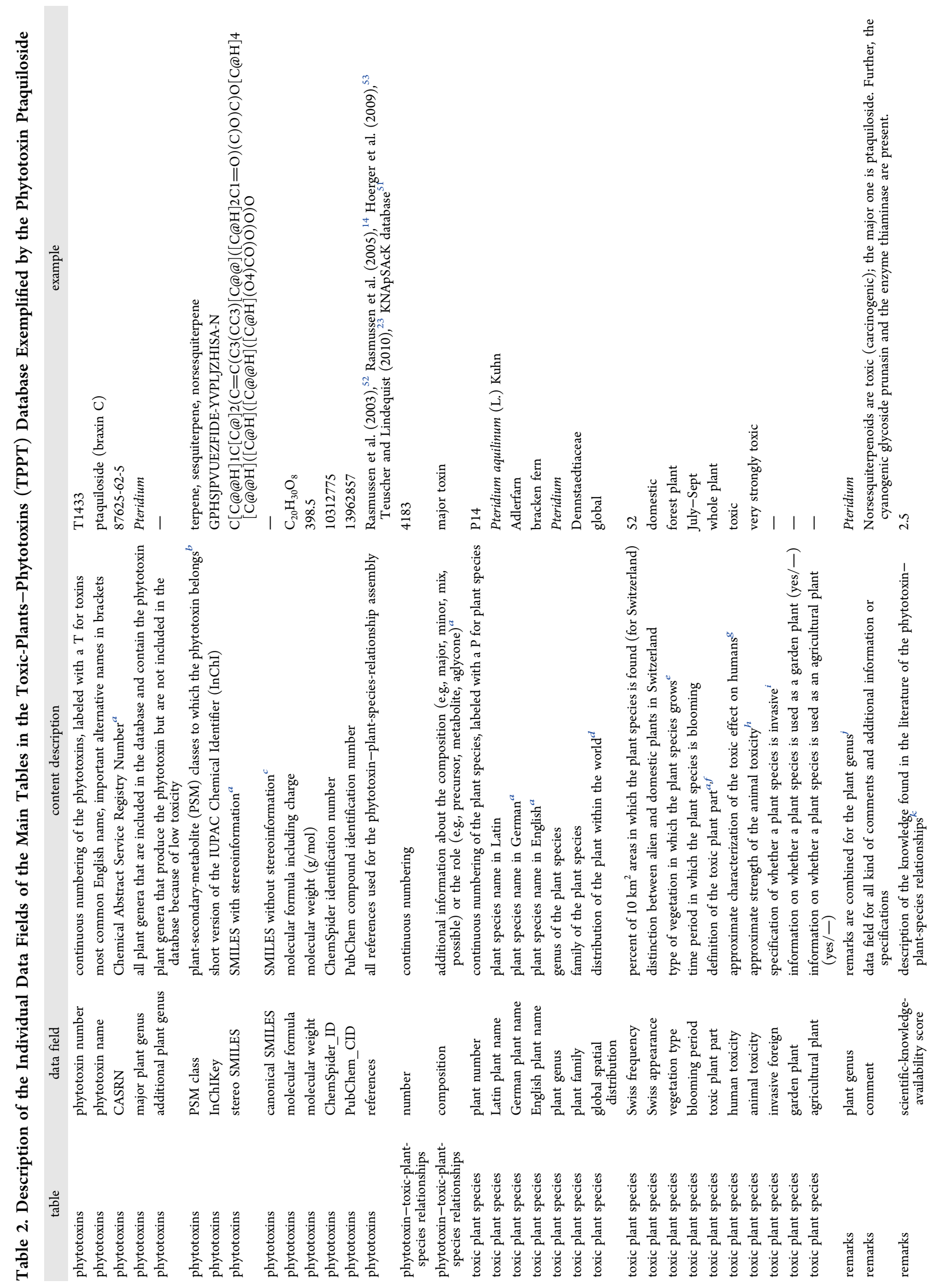




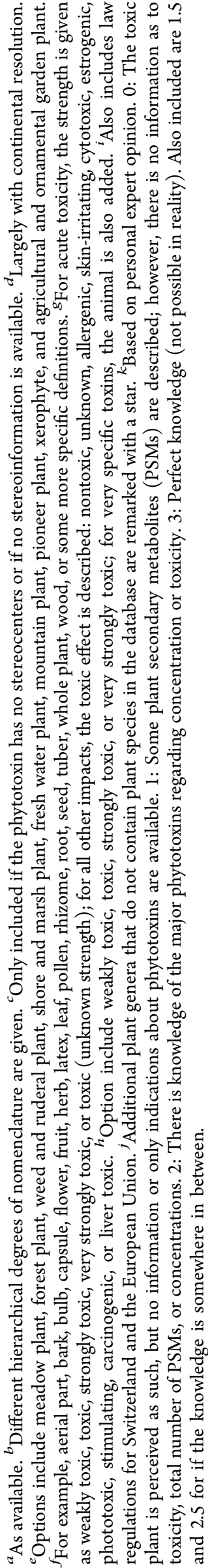

persistence $(\mathrm{P})$ and mobility $(\mathrm{M})$, and (iii) the acute rodent and the acute aquatic toxicity. A similar prioritization method was implemented for regulatory purposes by the German Environment Agency. Prior to the analysis, we removed ionic species and high MW substances $(>1000 \mathrm{~g} / \mathrm{mol})$, because they are not in the applicability domains of the predictions tools. Furthermore, substances were removed if no predictions were possible for a needed parameter. This resulted in 1506 substances included in the prioritization.

First, minimal phytotoxin occurrence is required as a prerequisite for their environmental importance. It was semiquantitatively approximated via the occurrence of the corresponding phytotoxinproducing plants. To this end, the Swiss-frequency parameter included in the TPPT database from Lauber et al., ${ }^{43}$ which encodes the distribution of a plant species in Switzerland, was used to define an occurrence factor and derive more general occurrence categories: no, very low, low, medium, and high occurrence (for technical details, see the SI).

Second, the environmental behavior was assessed by adapting the PM scoring procedure from Arp et al. in a simplified manner. ${ }^{6}$ The PM scoring uses the $D_{\mathrm{oc}}$, the $\mathrm{pH}$-dependent soil-organic-carbonwater partition coefficient $\left(K_{\mathrm{oc}}\right)$, as a measure of mobility, and the degradation half-life $\left(t_{1 / 2}\right)$ as a measure of the aquatic persistence (including biodegradation and hydrolysis). In this prioritization, we only used estimated properties because of a lack of experimental data. Furthermore, phototransformation was omitted because no good quantitative structure-activity relationships (QSARs) were available, and volatilization was excluded because it is not a true persistence parameter. ${ }^{6}$ The final classification distinguishes (i) the unproblematic compounds, including immobile compounds, unstable compounds, and transient compounds (immobile and unstable), from (ii) potentially relevant compounds, which are further classified in groups 1 to 5 with increasing importance (for technical details, see the SI).

Third, for the effect on mammalian species, the predicted and measured acute rodent toxicity $\left(\mathrm{LD}_{50}\right)$ was included, and for the effect on aquatic organisms, the predicted acute aquatic toxicity $\left(\mathrm{LC}_{50}\right.$ or the half-maximal effective concentration, $\mathrm{EC}_{50}$ ) was considered for the most sensitive species of fish, daphnia, and green algae. The substances were classified using the official system from the globally harmonized system of classification and labeling of chemicals (GHS; for details, see the SI).

All the phytotoxin information used in the prioritization is summarized in a combined table in the TPPT database, as defined in Table S6 in the SI. After performing the assessment for the individual phytotoxins, the phytotoxins were combined such that entire PSM classes were ranked according to the number of priority phytotoxins. Additionally, for all five parameters (i.e., phytotoxin occurrence, degradation half-life, $\log K_{\mathrm{oc}}$ or $\log D_{\mathrm{oc}}$ acute rodent toxicity, and acute aquatic toxicity), sensitivity and uncertainty analyses were performed. The sensitivity analysis was completed by individually varying the parameters by a factor of 2 and assessing the changes in the prioritization. For the uncertainty analysis, the derived uncertainty factors from Strempel et al. were adapted, ${ }^{5}$ and the minimum and maximum numbers of prioritized phytotoxins were determined.

\section{RESULTS AND DISCUSSION}

The TPPT database is a manually compiled and freely available database (downloadable from https://www.agroscope.admin. ch/agroscope/en/home/publications/apps/tppt.html) providing information about toxic plants in Central Europe and their phytotoxins. In total, 1586 phytotoxins produced by 844 plant species are included, which results in 6268 relationships between plant species and phytotoxins with a maximum of 30 phytotoxins per plant species. The strength of the TPPT database lies in the combination of the phytotoxin-plantspecies relationships with detailed biological and chemical information. 


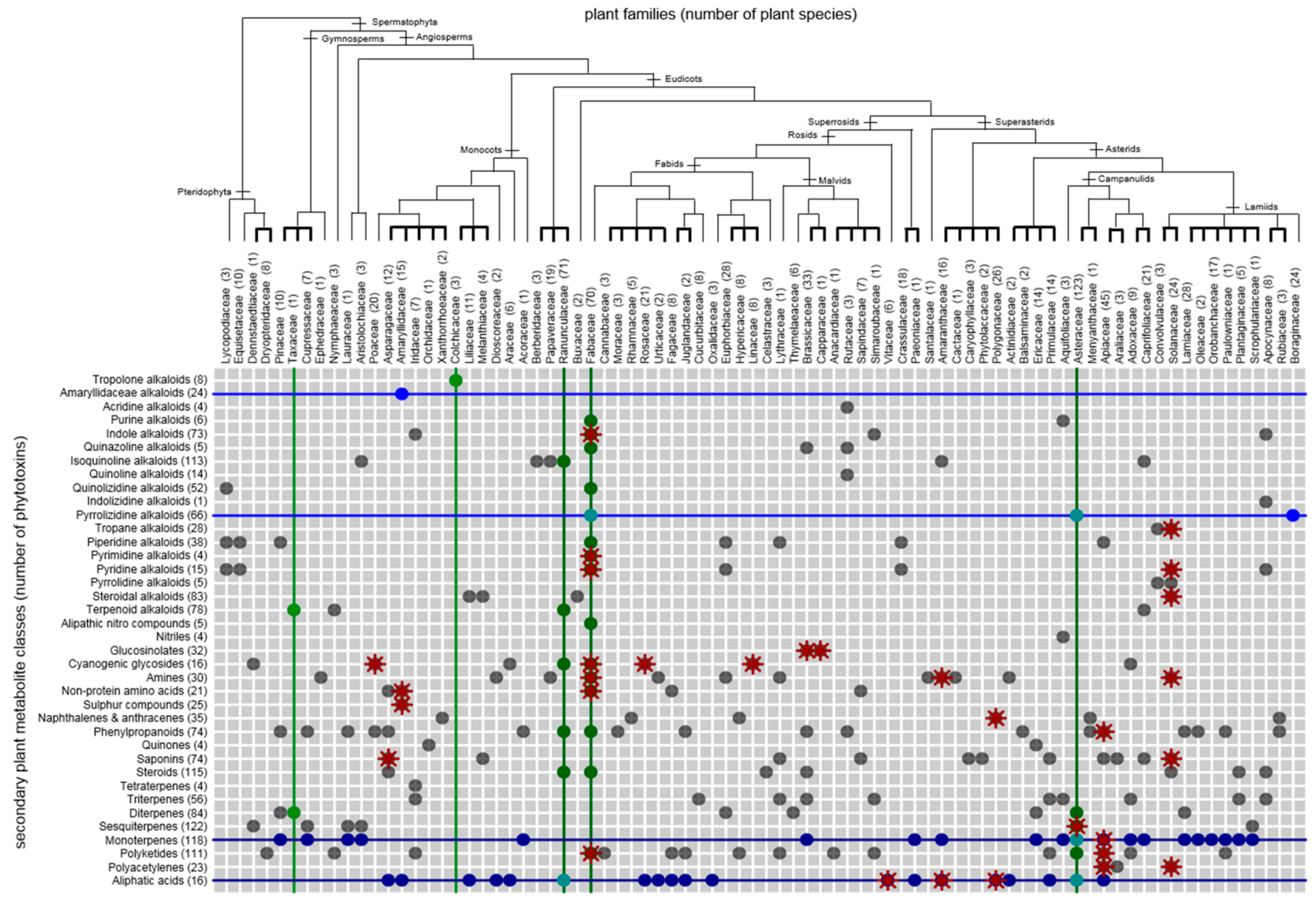

Figure 2. Distribution of the plant-secondary-metabolite (PSM) classes ( $y$-axis) in the different plant families ( $x$-axis). The brackets contain the number of phytotoxins for a PSM class and the number of plant species per plant family. In the genealogical tree, the thick branches combine the plant families into the systematic orders, for which the interrelationships are shown. ${ }^{48}$ The gray dots show all combinations; the blue, green, and turquoise dots and lines indicate the examples discussed in the main text; and the red stars indicate agricultural plant species.

TPPT Database: Toxic Plant Species. Of the total 844 plant species, 69 are agricultural plants, 176 are garden plants (ornamental garden plants and herbs), 689 occur naturally in Switzerland (105 are also garden plants), and 13 do not grow in Switzerland but were included because of their global agricultural importance. These numbers suggest that roughly $20 \%$ of the naturally growing plant species (over 3600) in Switzerland produce some PSMs with adverse effects. Among all plants, 241 are alien species in Switzerland, and of these, 70 plant species are invasive: 14 are of priority importance in the European Union, and 15 are prohibited in Switzerland (3 are on both concern lists). Interestingly, of the 26 most concerning plants, only 14 are ornamental garden plants that evaded into the environment, whereas 4 are weed and ruderal plants, and even more concerning in the present context, 8 are invasive water plants. Most of the invasive species are generally assumed to be nontoxic to humans, but their aquatic toxicity is still unknown, and for some of the invasive species their toxicity is entirely unknown. Overall, according to the categorization in Lauber et al., ${ }^{43}$ only $5.2 \%$ of the plants in the TPPT database are very strongly toxic, and $9.0 \%$ are strongly toxic, but considerably more plants, namely $37 \%$, are classified as medium toxic. The rest of the plant species are classified as weakly toxic (17\%), allergenic (12\%), toxic but not further classified (5.9\%), nontoxic (4.5\%), phototoxic $(4.1 \%)$, carcinogenic $(1.3 \%)$, unknown $(1.2 \%)$, liver toxic $(0.6 \%)$, estrogenic $(0.4 \%)$, stimulating $(0.4 \%)$, or cytotoxic $(0.1 \%)$. The classification according to specific modes of action, such as carcinogenicity or liver toxicity, is not complete because many plants have not been tested systematically for them. Of all included toxic plant species, $46 \%$ occur in less than $10 \%$ of the area of Switzerland and only $15 \%$ occur in more than $50 \%$, which is a consequence of the highly diverse vegetation zones in this country. The TPPT database contains additional biological information, such as the vegetation type, the blooming period, and the toxic plant part, which can be used to set up dedicated environmental-monitoring networks.

TPPT Database: Phytotoxins. For all plant species, 1586 phytotoxins were identified and classified into the different PSM classes, as indicated in Figure 2. The highest number of toxins is found for alkaloids and terpenes, which prevail among all PSMs, making up between 60 and 70\%. ${ }^{9}$ PSM classes with intermediate phytotoxin numbers are the steroids, saponins, phenylpropanoids, and polyketides. Steroids and saponins are glycosylated structures with mostly higher molecular weights, and parts of the structures are also synthesized from isoprene units such as the terpenes. Phenylpropanoids and polyketides are more diverse classes, including, among others, coumarins and lignans in the former and flavones, cannabinoids, and catechins in the latter. Several PSM classes only contain small numbers of individual substances, such as the glucosinolates or the cyanogenic compounds. Although the physicochemical 
properties within a PSM class are often similar, these properties cover a broad range across all classes: the molecular weights range between 31 and $1968 \mathrm{~g} / \mathrm{mol}$ and are most often between 300 and $400 \mathrm{~g} / \mathrm{mol}$, the in silico estimated $\log K_{\text {ow }}$ values range between -10.45 and 13.59 (which goes beyond the applicability domain of $\mathrm{KOWWIN}^{38}$ ) with an average of 1.89 , and the numbers of functional groups also vary with up to 26 hydrogen-bond donors and up to 49 hydrogen-bond acceptors. The corresponding distribution curves are shown in Figure S1. Finally, it should be noted that the absolute number of phytotoxins in a PSM class is no indicator of their overall environmental risk, but rather the physicochemical and toxicological properties of the different PSM classes are relevant, as discussed in the case study below.

TPPT Database: Phytotoxin-Toxic-Plant-Species Relationships. Figure 2 assigns the different PSM classes to the taxonomic plant families, showing that the prevalences within plant families vary strongly for different PSM classes. Whereas some PSM classes have broad distributions, such as those of the aliphatic acids or monoterpenes (Figure 2, dark blue lines), other classes are limited to a small number of plant families, as it is the case for most alkaloids (e.g., Amaryllidaceae alkaloids or pyrrolizidine alkaloids; Figure 2, light blue lines). A broad distribution might be attributed to either an early phylogenetic development, or to a convergence in plant evolution. Detailed discussions of the evolution of PSMs and their distribution in different plants are given elsewhere, for example, by Wink. ${ }^{9}$

Asteraceae, Ranunculaceae, and Fabaceae are plant families with very high numbers of toxic plant species (over 50 species per plant family; Figure 2, dark green lines). However, whereas the Fabaceae have a large variability of PSM classes (Figure 2), the Asteraceae and Ranunculaceae contain only small numbers of PSM classes. For instance, the Asteraceae produce mostly pyrrolizidine alkaloids and terpenes. The other extreme includes the plant families with only a few toxic plant species, but several of them are very important due to their high toxicity. For example, the English yew (Taxus baccata, Taxacaceae; Figure 2, light green) is often grown in gardens, and the autumn crocus (Colchicum autumnale, Colchicaceae; Figure 2, light green) is problematic because of confusion with wild garlic (Allium ursinum, Amaryllidaceae). Overall, for only approximately $10 \%$ of the plants, no data on the occurrence of toxic PSMs are available. For the majority of the PSM classes with adverse effects, some phytotoxins are known, although the number of known phytotoxins may still vary. Figure 2 also demonstrates the high PSM-class variability in agricultural plants (Figure 2, red stars): there are steroidal alkaloids in potato; pyridine alkaloids in tobacco; monoterpenes and phenylpropanoids in many herbs, including dill, coriander, and parsley; glucosinolates in cabbages; and cyanogenic glycosides in many fruits, including apple and peach.

TPPT Database: Limitations. We compiled the TPPT database to the best of our knowledge; however, it is practically impossible to generate a complete database, and expert judgements were needed in case of contradictory data. Regarding the phytotoxin-toxic-plant-species relationships, a false-positive uncertainty exists because of the possible inclusion of less relevant phytotoxins, and a false-negative uncertainty exists as a result of the absence of information about not-yet-recognized but relevant phytotoxins. Several causes add to these uncertainties, including limited data availability, conflicting literature data, and biological variations such as spatial differences or subspecies. To account for these uncertainties, we included a comment field and a scientificknowledge-availability score, reflecting the number of references for a certain plant genus, and some data fields were even left blank. Finally, the TPPT database can be updated and extended whenever new research data are available.

Although much information is included in the TPPT database, a remarkable part of it, in particular the phytotoxins' physicochemical properties and toxicity, is in silico estimations and not (yet) experimentally measured data. These estimations provide the first approximations of the properties and some rather good calculations (e.g., ptaquiloside has a predicted log $K_{\text {ow }}$ of -0.95 and a measured $\log K_{\text {ow }}$ of -0.68$),{ }^{14}$ but they need to be evaluated critically. Compared with typical anthropogenic substances, phytotoxins often have higher molecular weights and are more ionizable with more functional groups, which also indicates that they are underrepresented in QSAR-calibration data sets. Therefore, two prerequisites are required before QSAR estimations can reliably be performed for phytotoxins: first, measurements need to be performed to generate experimental data, and second, the QSAR applicability domain needs to be expanded and validated using the experimental data. For now, the implementation of these prerequisites would exceed the scope of this paper, but they are necessary for more in-depth environmental risk assessments.

Assessment of Phytotoxins with Respect to Their PMT Characteristics. For the assessment of the phytotoxins' PMT characteristics, four primary properties were taken into consideration: the shortest degradation half-life, the $\mathrm{pH}$ dependent $K_{\mathrm{oc}}$, the acute rodent toxicity, and the acute aquatic toxicity. The distributions of these properties, shown in Figure 3, largely follow those of anthropogenic chemicals, as presented, for example, by Strempel et al. ${ }^{5}$ Differences exist for the $\mathrm{pH}$-dependent $K_{\mathrm{oc}}$ (Figure $3 \mathrm{~b}$ ), which tends to be higher, and the half-life (Figure 3a), where it becomes visible that the phytotoxins generally have higher degradability. Most probably, the persistence would even further decrease if a more appropriate QSAR for hydrolysis half-life estimations were available. The half-life is also the most sensitive property, as indicated by the dark shaded area in Figure 3a, but the toxicity predictions are much more uncertain despite low sensitivities (Figure 3c,d).

For the assessment of the environmental behavior, the phytotoxins were classified according to the PM characteristics from Arp et al., ${ }^{6}$ and the resulting classification of the phytotoxins in terms of aquatic persistence and mobility is shown in Figure 4a,b, respectively. The classified aquatic persistence is either relatively high (P4) or low (P1), for which the major degradation mechanism is biodegradation, accounting for over $98 \%$ of the shortest half-lives. Regarding the mobility, the classification provides evidence that most phytotoxins are mobile in the environment, with over 1000 compounds in the most mobile (M5) category. This reflects the generally high polarity of PSMs and the corresponding low number of nonionizable compounds (39\%). Even several terpene classes with a generally low water solubility are mobile in the environment because of the high number of oxygen atoms. The classification in the combined PM score is shown in Figure 4c. The environmentally less relevant categories (transient, unstable, and immobile) make up almost $37 \%$ as a result of the high number of rapidly degradable phytotoxins. Including the uncertainty analysis, the values range between 17 and $65 \%$, which is a broad range and the result of a very sensitive degradation half-life, as indicated in Figure $3 \mathrm{~b}$. 

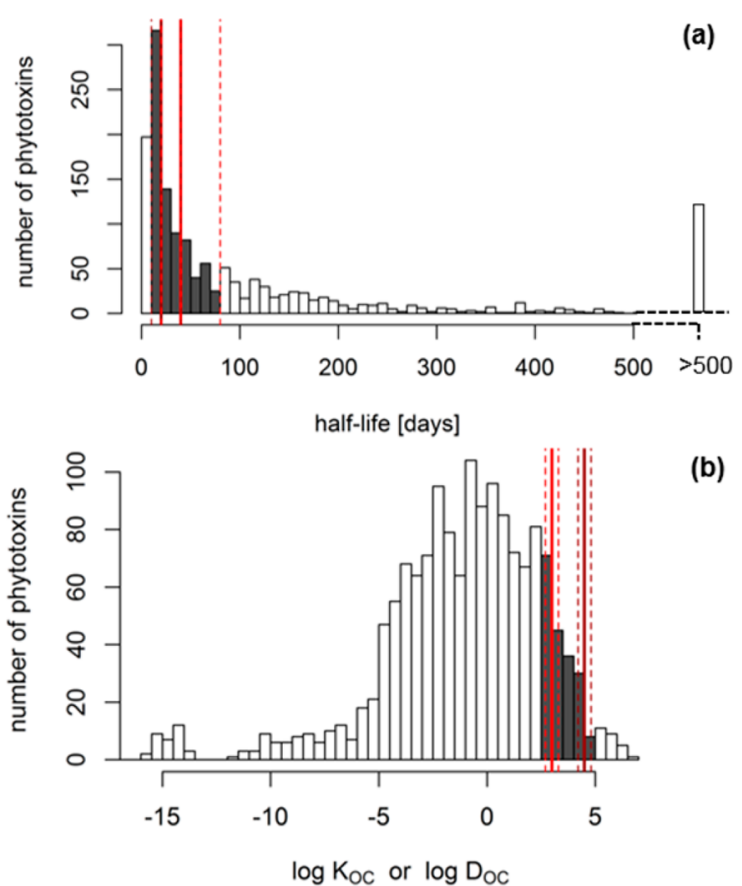

(b)
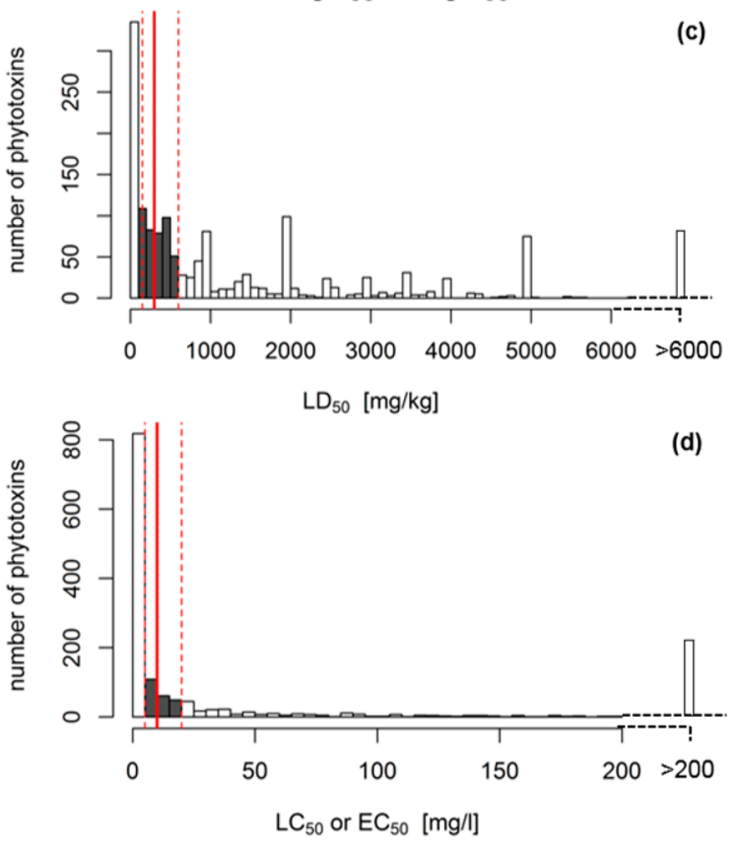

Figure 3. Distribution and sensitivities of the included phytotoxins within the four primary PMT properties: (a) degradation half-life, (b) soil-organic-carbon-water-partition coefficient $\left(K_{\mathrm{oc}}\right)$ or $\mathrm{pH}$-dependent soil-organic-carbon-water-partition coefficient $\left(D_{\mathrm{oc}}\right),(\mathrm{c})$ acute rodent toxicity (median lethal dose, $\mathrm{LD}_{50}$ ), and (d) acute aquatic toxicity (lethal median concentration, $\mathrm{LC}_{50}$, or half-maximal effective concentration, $\left.\mathrm{EC}_{50}\right)$. In each panel, the red line indicates the threshold in the prioritization procedure, the dashed lines indicate the sensitivity range defined by a factor of 2 around the threshold, and the filled columns point out the affected range of the data. For $(a, b)$, two thresholds are included, a more cautious dark-red one and a less cautious brighter-red one.

However, phytotoxins with high production and release might still accumulate and as such maintain relatively high steadystate concentrations. Consistently, the most critical score, PM5, accounts for 40\%, ranging between 15 and $68 \%$, including the uncertainties. The classification largely reflects the $\mathrm{P}$ score, whereas the $\mathrm{M}$ score has a minor influence because most of the compounds are mobile. The estimation of degradation half-lives should therefore be evaluated even more critically. This classification is also in accordance with the results from Arp et al. in the analysis of anthropogenic compounds. ${ }^{6}$ Although this is a preliminary analysis with high uncertainties, the high fraction of prioritized phytotoxins should raise concerns about their impact on the aquatic environment. The most critical PSM classes in terms of environmental behavior include several alkaloid classes, steroids, and saponins (see Table S9). Other PSM classes have more varying properties, but diterpenes, triterpenes, sesquiterpenes, polyketides, and naphthalene- and anthracenederivatives might also be potentially relevant. Generally, of less importance are aliphatic acids, cyanogenic glycosides, polyacetylenes, nonprotein amino acids and amines, glucosinolates, phenylpropanoids, sulfur compounds, monoterpenes, and other small-molecular-weight alkaloids, which mostly degrade fast.

To assess the impacts on organisms, the toxicities of the phytotoxins were assessed. The rodent and aquatic toxicities were evaluated on the basis of the classes of the GHS and were shown to be uncorrelated to each other (see Figure S3). The acute rodent toxicity, based on measured and predicted ProTox data, showed the following classification according to GHS for acute toxicity to humans: $18,41,18,12$, and $5 \%$ in GHS classes 5, 4, 3, 2, and 1 (with increasing toxicity) respectively; $6 \%$ were nontoxic according GHS categorization (Figure S2a). This classification is also reflected by the ProTox training set. ${ }^{41}$ Interestingly, only a small fraction of the toxins are strongly toxic, but over $40 \%$ are only in category 4 . By far the most toxic PSM class seems to be steroids, followed by certain alkaloids and saponins (see Table S9). For the aquatic toxicity, a different distribution into the GHS classes for substances with acute hazard to the aquatic environment was found: $20 \%$ were in the least toxic GHS class, $3 ; 22 \%$ were in GHS class 2 ; $39 \%$ were in the most toxic GHS class, 1; and $20 \%$ were predicted to be non-ecotoxic (Figure S2b). Here, a major portion of the phytotoxins are classified in the worst category and are therefore highly relevant. Overall, green algae were predicted to be most sensitive, accounting for $53 \%$ of the lowest toxicities, followed by daphnia (26\%) and fish (21\%). The most ecotoxic classes are again alkaloids but also several terpenes, polyacetylenes, phenylpropanoids, and polyketides, whereas the steroids and saponins seem to be less critical compared with the acute rodent toxicity (see Table S9).

Prioritization of Phytotoxins According to Their Aquatic Micropollution Potentials and Identification of the Most Critical PSM Classes. Finally, we combined the prerequisite of a general phytotoxin occurrence with the PM scoring and toxicity in a simple qualitative risk assessment to identify the most critical PSM classes for the aquatic environment. A prioritization procedure was performed by setting a limit for each of the three characteristics, as shown in Figure 5. The phytotoxin occurrence was taken as the first prioritization step, because the general occurrence is a prerequisite for any micropollution potential, and phytotoxins with medium or high occurrence ( $65 \%$ in total) were regarded as potentially the most relevant (more details are found in the SI). Therefore, 526 phytotoxins were removed in this step. For now, we used the plant distribution in Switzerland to derive the phytotoxins' occurrence, but for a more in-depth assessment the plant abundance and phytotoxin concentrations 

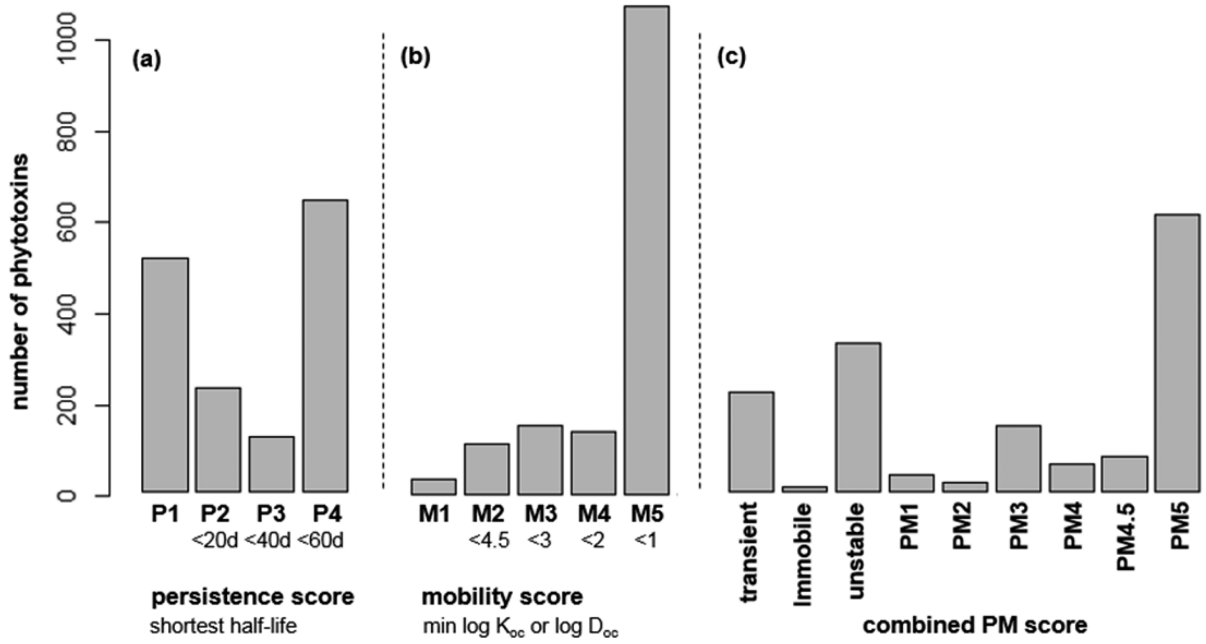

Figure 4. Classification of phytotoxins according to (a) aquatic persistence (P) score, (b) mobility (M) score, and (c) combined PM score based on Arp et al. ${ }^{6}$ The phytotoxins are categorized with increasing importance according to the shortest half-life (given in days) for aquatic persistence and to the minimal logarithmic soil-organic-carbon-water-partition coefficient $\left(\log K_{\mathrm{oc}}\right)$ or the $\mathrm{pH}$-dependent soil-organic-carbon-water-partition coefficient $\left(\log D_{\mathrm{oc}}\right)$ for the mobility. The combined PM scores differentiate transient, immobile, unstable, and potential aquatic micropollutants with increasing importance (from 1 to 5 ).

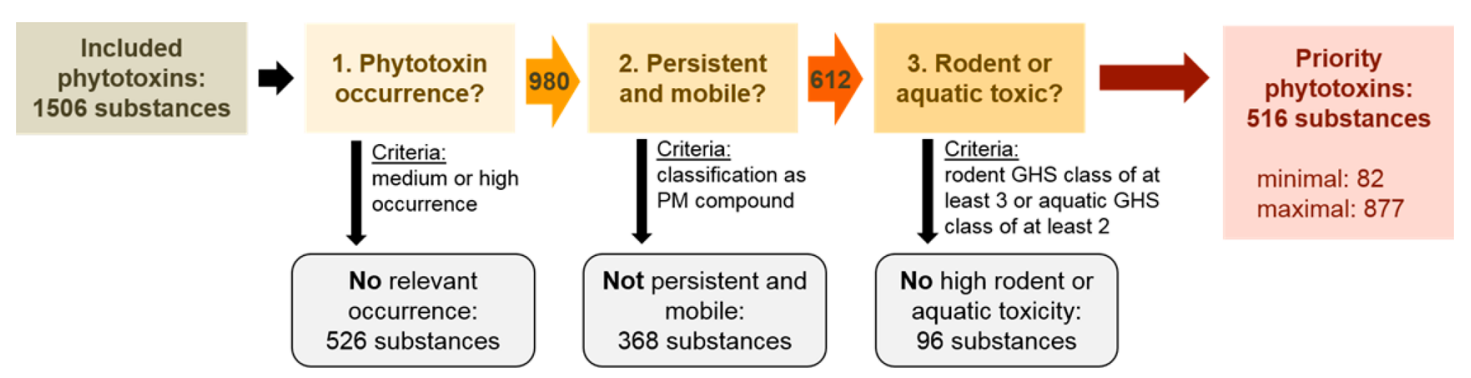

Figure 5. Scheme of the prioritization procedure combining phytotoxin occurrence, environmental behavior, and toxicity to identify phytotoxins of possible relevance for the safety of the aquatic environment in Central Europe. The number of excluded phytotoxins in each step is stated together with the starting number and the prioritized number. For details, see the text.

in the plants would be necessary. Furthermore, rarely occurring phytotoxins might also pose a local risk in plant hot-spots and catchment areas without much dilution and should not be fully ignored in more extensive assessments. In the second prioritization step, all phytotoxins were removed that were either not persistent or not mobile (i.e., were not classified into a PM category), which included 368 phytotoxins. And in the last step, 96 phytotoxins were removed, because they were neither harmful in terms of acute rodent toxicity (GHS category 4 or less) nor ecotoxic (GHS category 3 or less). On this basis, 516 phytotoxins of priority remained; taking the uncertainty into account resulted in a minimum of 82 phytotoxins and a maximum of 877 phytotoxins. This high uncertainty is caused by the very sensitive half-life estimations and the uncertain toxicity estimations (see above). The average number of prioritized phytotoxins corresponds to over $34 \%$ of all phytotoxins in the TPPT database and is a remarkably high number. It should be kept in mind that the prioritization is mainly based on predicted properties, and only a subset of all worldwide produced phytotoxins is included. However, even if only a small fraction of the potentially relevant phytotoxins are actually present in the aquatic environment, they might sum up and pose a risk.

Table 3 ranks PSM classes according to the percentage of individual plant toxins prioritized in the above procedure (a complete list is presented in Table S10). It clearly shows that alkaloids; terpenes, including steroids and saponins; and partially polyketides are potentially the most relevant for the aquatic environment. These PSM classes contain high numbers of toxic, frequently produced, mobile, and persistent phytotoxins, which are important to consider in monitoring programs and risk assessments. Several PSM classes are supposed to be unproblematic, either because of low production by local plants or fast degradation in the environment, which applies, for instance, to cyanogenic glycosides and glucosinolates that are often produced by agricultural plants.

To evaluate the prioritization, we analyzed the classification of some phytotoxins for which one or several environmental case studies were found in the literature. Rasmussen et al. analyzed the environmental behavior of the carcinogenic ptaquiloside and concluded that this norsesquiterpene could possibly leach into the aquatic environment depending on soil type, temperature, and most crucially the $\mathrm{pH}^{44}$ Correctly, this phytotoxin was prioritized in the present risk assessment. Artemisinin is another prioritized sesquiterpene which is further used as an antimalaria drug and for which the detected soil concentrations were found to be in the range of toxic-effect concentrations. ${ }^{45}$ Also prioritized were estrogenic isoflavones; for example, formononetin was regularly detected in the drainage water of a red clover field and even in river waters. ${ }^{13}$ An example of an unprioritized phytotoxin is juglone, which is 
Table 3. Ranking of the Potentially Relevant PlantSecondary-Metabolite (PSM) Classes According to the Number of Priority Phytotoxins That Were Identified by the Procedure Described in the Text ${ }^{a}$

\begin{tabular}{|c|c|c|c|}
\hline PSM class & $\begin{array}{l}\text { total } \\
\text { phytotoxins } \\
\text { included }\end{array}$ & $\begin{array}{l}\text { prioritized } \\
\text { phytotoxins }\end{array}$ & examples \\
\hline steroids & 115 & 56 & $\begin{array}{l}\text { digitoxigenin, } \\
\text { strophanthidin }\end{array}$ \\
\hline $\begin{array}{l}\text { terpenoid } \\
\text { alkaloids }\end{array}$ & 77 & 52 & aconitine, taxine B \\
\hline $\begin{array}{l}\text { pyrrolizidine } \\
\text { alkaloids }\end{array}$ & 65 & 48 & $\begin{array}{l}\text { lycopsamine, } \\
\text { heliosupine }\end{array}$ \\
\hline steroidal alkaloids & 81 & 45 & $\begin{array}{l}\text { protoveratrine } \mathrm{A} \text {, } \\
\text { cyclobuxine } \mathrm{D}\end{array}$ \\
\hline $\begin{array}{l}\text { isoquinoline } \\
\text { alkaloids }\end{array}$ & 89 & 45 & protopine \\
\hline $\begin{array}{l}\text { quinolizidine } \\
\text { alkaloids }\end{array}$ & 52 & 34 & lupanine, sparteine \\
\hline sesquiterpenes & 122 & 31 & ptaquiloside, artabsin \\
\hline polyketides & 110 & 30 & $\begin{array}{l}\text { formononetin, } \\
\text { lupulone }\end{array}$ \\
\hline indole alkaloids & 72 & 27 & vincamine \\
\hline triterpenes & 56 & 26 & $\begin{array}{l}\text { cucurbitacin B, } \\
\text { elaterinide }\end{array}$ \\
\hline diterpenes & 83 & 26 & $\begin{array}{l}\text { baccatin III, } \\
\text { mezerein }\end{array}$ \\
\hline $\begin{array}{l}\text { Amaryllidaceae } \\
\text { alkaloids }\end{array}$ & 23 & 19 & $\begin{array}{l}\text { galanthamine, } \\
\text { lycorine }\end{array}$ \\
\hline \multicolumn{4}{|c|}{$\begin{array}{l}{ }^{a} \text { Examples of specific phytotoxins are also given. A table with all PSM } \\
\text { classes and a figure of all exemplified chemical structures are given in } \\
\text { Table S10 and Figure S5, respectively. }\end{array}$} \\
\hline
\end{tabular}

a toxic napthoquinone (phenylpropanoid) produced by walnut trees. Von Kiparski et al. found that juglone is microbially and abiotically degradable and actually quite short-lived in soils with microbial activity, which is consistent with the estimated biodegradation half-life of $12 \mathrm{~h} .{ }^{46}$ However, juglone was found in soils beneath walnut trees and von Kiparski et al. concluded that juglone can still accumulate in soils with low microbial activity because of the high amounts released. ${ }^{46}$ Correctly, juglone is not prioritized here because the degradation in water is usually even faster and no accumulation is possible. The two major potato plant glycoalkaloids, $\alpha$-solanine and $\alpha$-chaconine (steroidal alkaloids), were also not prioritized, which is in accordance with the results found by Jensen et al. ${ }^{12}$ Their results indicated low leaching potential because no glycoalkaloids were detected in the groundwater despite concentrations of up to $25 \mathrm{~kg} / \mathrm{ha}$ in the plants themselves. However, these two glycoalkaloids were excluded in the prioritization procedure because of low occurrence, but they were classified in the highest PM category (PM 5), which indicates an erroneous PM classification. In fact, in silico estimations for higher-molecular-weight compounds with several sugar units are generally not very precise and are probably outside the application domain. However, for many PSM classes, no environmental-behavior studies were found, particularly for many important PSM classes, such as most alkaloids.

Although the above examples illustrate the general plausibility of the proposed prioritization procedure and also its limitations, its actual predictive power remains to be evaluated with monitoring campaigns that include plant toxins. For the time being, several PSM classes could be identified for which further investigations should be done, and this work provides a starting point for this endeavor. Overall, the TPPT database was shown to be useful for preliminary risk assessments. Furthermore, the TPPT database is expected to be of value in other fields dealing with toxic plants and their toxins and might be applied by environmental scientists, food scientists, veterinary scientists, biologists, or toxicologists. For example, in the Marie Skłodowska-Curie Innovative Training Network NaToxAq, which focuses on natural toxins in the aquatic environment, the TPPT database is applied in the phytotoxin investigations. ${ }^{47}$ To conclude, we would like to emphasize the necessity for further research on phytotoxins in the environment because we found over $34 \%$ to be potential aquatic micropollutants. There is a need for, on the one hand, better estimations and more measured property data to enable more precise estimations and, on the other hand, monitoring programs actually including phytotoxins as target or suspect analytes.

\section{ASSOCIATED CONTENT}

\section{Supporting Information}

The Supporting Information is available free of charge on the ACS Publications website at DOI: 10.1021/acs.jafc.8b01639.

Methodological details on data compilation, measured toxicity data, in silico physicochemical-property estimations (EPI Suite and ACD/Percepta), in silico rodenttoxicity prediction (ProTox), in silico aquatic toxicity predictions (ECOSAR), occurrence analysis, PMT analysis, sensitivity and uncertainty analysis, and descriptions of all data fields in the TPPT database; further results including distributions of different physicochemical properties among all phytotoxins, distributions of phytotoxins in the occurrence and toxicity categories, comparison of the acute rodent toxicity and the acute aquatic toxicity, details on the sensitivity and uncertainty analyses, characterization of PSM classes and resulting prioritization, and chemical structures of phytotoxins discussed in the main text (PDF)

TPPT database (XLSX)

\section{AUTHOR INFORMATION}

\section{Corresponding Author}

*Tel.: 0414437773 42. E-mail: thomas.bucheli@agroscope. admin.ch.

\section{ORCID}

Barbara F. Günthardt: 0000-0003-0319-5272

Juliane Hollender: 0000-0002-4660-274X

Martin Scheringer: 0000-0002-0809-7826

Thomas D. Bucheli: 0000-0001-9971-3104

Funding

Funding from the Swiss National Science Foundation (Project "PHYtotoxins: aquatic miCROPOLLutants of concern?" (PHYCROPOLL); grant no. 200021_162513/1) is gratefully acknowledged.

\section{Notes}

The authors declare no competing financial interest.

\section{ACKNOWLEDGMENTS}

Special thanks go to Carina D. Schönsee, Daniela Rechsteiner, and Inés Rodriguez Leal for many fruitful discussions. 


\section{REFERENCES}

(1) Moschet, C.; Wittmer, I.; Simovic, J.; Junghans, M.; Piazzoli, A.; Singer, H.; Stamm, C.; Leu, C.; Hollender, J. How a complete pesticide screening changes the assessment of surface water quality. Environ. Sci. Technol. 2014, 48, 5423.

(2) Verlicchi, P.; Al Aukidy, M.; Zambello, E. Occurrence of pharmaceutical compounds in urban wastewater: Removal, mass load and environmental risk after a secondary treatment-A review. Sci. Total Environ. 2012, 429, 123-155.

(3) Kasprzyk-Hordern, B.; Dinsdale, R. M.; Guwy, A. J. The occurrence of pharmaceuticals, personal care products, endocrine disruptors and illicit drugs in surface water in South Wales, UK. Water Res. 2008, 42, 3498-3518.

(4) Howard, P. H.; Muir, D. C. G. Identifying new persistent and bioaccumulative organics among chemicals in commerce. Environ. Sci. Technol. 2010, 44, 2277.

(5) Strempel, S.; Scheringer, M.; Ng, C. A.; Hungerbühler, K. Screening for PBT Chemicals among the "Existing" and "New" Chemicals of the EU. Environ. Sci. Technol. 2012, 46, 5680-5687.

(6) Arp, H. P. H.; Brown, T. N.; Berger, U.; Hale, S. E. Ranking REACH registered neutral, ionizable and ionic organic chemicals based on their aquatic persistency and mobility. Environ. Sci.Proc. Imp. 2017, 19, 939-955.

(7) Neumann, M.; Schliebner, I. Protecting the sources of our drinking water; German Environment Agency: Dessau-Rosslau, 2017.

(8) Reemtsma, T.; Berger, U.; Arp, H. P. H.; Gallard, H.; Knepper, T. P.; Neumann, M.; Quintana, J. B.; Voogt, P. d. Mind the Gap: Persistent and Mobile Organic Compounds-Water Contaminants That Slip Through. Environ. Sci. Technol. 2016, 50, 10308-10315.

(9) Wink, M. Evolution of secondary metabolites from an ecological and molecular phylogenetic perspective. Phytochemistry 2003, 64, 319.

(10) Wittstock, U.; Gershenzon, J. Constitutive plant toxins and their role in defense against herbivores and pathogens. Curr. Opin. Plant Biol. 2002, 5, 300-307.

(11) Bucheli, T. D. Phytotoxins: Environmental Micropollutants of Concern? Environ. Sci. Technol. 2014, 48, 13027-13033.

(12) Jensen, P. H.; Strobel, B. W.; Hansen, H. C. B.; Jacobsen, O. S. Fate of toxic potato glycoalkaloids in a potato field. J. Agric. Food Chem. 2009, 57, 2862-2867.

(13) Hoerger, C. C.; Wettstein, F. E.; Bachmann, H. J.; Hungerbuehler, K.; Bucheli, T. D. Occurrence and Mass Balance of Isoflavones on an Experimental Grassland Field. Environ. Sci. Technol. 2011, 45, 6752-6760.

(14) Rasmussen, L. H.; Hansen, H. C. B.; Lauren, D. Sorption, degradation and mobility of ptaquiloside, a carcinogenic bracken (Pteridium sp.) constituent, in the soil environment. Chemosphere 2005, 58, 823-835.

(15) Bucheli, T. D.; Strobel, B. W.; Hansen, H. C. B. Personal Care Products Are Only One of Many Exposure Routes of Natural Toxic Substances to Humans and the Environment. Cosmetics 2018, 5, 10.

(16) Schmidt, U.; Struck, S.; Gruening, B.; Hossbach, J.; Jaeger, I. S.; Parol, R.; Lindequist, U.; Teuscher, E.; Preissner, R. SuperToxic: A comprehensive database of toxic compounds. Nucleic Acids Res. 2009, 37, D295-D299.

(17) Banerjee, P.; Erehman, J.; Gohlke, B.-O.; Wilhelm, T.; Preissner, R.; Dunkel, M. Super Natural II-a database of natural products. Nucleic Acids Res. 2015, 43, D935-D939.

(18) Judson, R.; Richard, A.; Dix, D.; Houck, K.; Elloumi, F.; Martin, M.; Cathey, T.; Transue, T. R.; Spencer, R.; Wolf, M. ACToR Aggregated Computational Toxicology Resource. Toxicol. Appl. Pharmacol. 2008, 233, 7-13.

(19) Takahashi, H.; Hirai, A.; Shojo, M.; Matsuda, K.; Parvin, A. K.; Asahi, H.; Nakamura, K.; Altaf-Ul-Amin, M.; Kanaya, S. SpeciesMetabolite Relation Database KNApSAcK and Its Multifaceted Retrieval System, KNApSAcK Family. In General, Applied and Systems Toxicology; John Wiley \& Sons: Chichester, U.K., 2011.

(20) European Food Safety Authority. Compendium of botanicals reported to contain naturally occuring substances of possible concern for human health when used in food and food supplements. EFSA J. 2012, 10, 2663

(21) Clinical Toxicology (CliniTox). http://www.vetpharm.uzh.ch/ clinitox/ (accessed Oct 20, 2017).

(22) Burrows, G. E.; Tyrl, R. J. Toxic Plants of North America, 2nd ed.; Wiley-Blackwell: Ames, IA, 2013.

(23) Teuscher, E.; Lindequist, U. Biogene Gifte: Biologie-ChemiePharmakologie-Toxikologie, 3rd ed.; Wissenschaftliche Verlagsgesellschaft mbH: Stuttgart, Germany, 2010.

(24) Roth, L.; Daunderer, M.; Komann, K. Giftpflanzen-Pflanzengifte: Vorkommen, Wirkung, Therapie, allergische und phototoxische Reaktionen, 6th ed.; Nikol: Hamburg, Germany, 2012.

(25) Quattrocchi, U. CRC World Dictionary of Medicinal and Poisonous Plants: Common Names, Scientific Names, Eponyms, Synonyms, and Etymology; CRC Press: Boca Raton, FL, 2012.

(26) Ellenberger, H.; Leuschner, C. Vegetation Mitteleuropas mit den Alpen: in ökologischer, dynamischer und historischer Sicht, 6th ed.; Eugen Ulmer: Stuttgart, 2010.

(27) Federal Office for the Environment (FOEN). Zustand der Artenvielfalt in der Schweiz. https://www.bafu.admin.ch/bafu/de/ home/themen/biodiversitaet/fachinformationen/zustand-derbiodiversitaet-in-der-schweiz/zustand-der-artenvielfalt-in-der-schweiz. html (accessed Oct 25, 2017).

(28) Info Flora: The National Data and Information Center on the Swiss Flora. https://www.infoflora.ch/en/ (accessed Oct 20, 2017).

(29) Buratti, F. M.; Manganelli, M.; Vichi, S.; Stefanelli, M.; Scardala, S.; Testai, E.; Funari, E. Cyanotoxins: producing organisms, occurrence, toxicity, mechanism of action and human health toxicological risk evaluation. Arch. Toxicol. 2017, 91, 1049-1130.

(30) Singh, S.; Kate, B. N.; Banerjee, U. Bioactive compounds from cyanobacteria and microalgae: an overview. Crit. Rev. Biotechnol. 2005, $25,73-95$.

(31) Gutzeit, H. O.; Ludwig-Müller, J. Plant Natural Products: Synthesis, Biological Function and Practical Applications, 1st ed.; WileyVCH: Weinheim, Germany, 2014.

(32) Poutaraud, A.; Michelot-Antalik, A.; Plantureux, S. Grasslands: A Source of Secondary Metabolites for Livestock Health. J. Agric. Food Chem. 2017, 65, 6535-6553.

(33) Kim, S.; Thiessen, P. A.; Bolton, E. E.; Chen, J.; Fu, G.; Gindulyte, A.; Han, L.; He, J.; He, S.; Shoemaker, B. A.; Wang, J.; Yu, B.; Zhang, J.; Bryant, S. H. PubChem. Substance and Compound databases. https://pubchem.ncbi.nlm.nih.gov/ (accessed Dec 6, 2016).

(34) ChemSpider. http://www.chemspider.com/ (accessed Dec 6, 2017).

(35) SQLite. https://sqlite.org/index.html (accessed Oct 19, 2017).

(36) Müller, K.; Wickham, H.; James, D. A.; Falcon, S. RSQLite: 'SQLite' Interface for $R$. https://CRAN.R-project.org/package $=$ RSQLite (accessed Oct 19, 2017).

(37) Wickham, H.; Müller, K. DBI: R Database Interface. https:// CRAN.R-project.org/package=DBI (accessed Oct 19, 2017).

(38) Estimation Programs Interface Suite for Microsoft Windows, v. 4.11; United States Environmental Protection Agency: Washington, DC, 2017.

(39) Gouin, T.; Cousins, I.; Mackay, D. Comparison of two methods for obtaining degradation half-lives. Chemosphere 2004, 56, 531-535.

(40) ACD/Percepta, release 2016.2; Advanced Chemistry Development, Inc.: Toronto, Canada, 2016.

(41) Drwal, M. N.; Banerjee, P.; Dunkel, M.; Wettig, M. R.; Preissner, R. ProTox: a web server for the in silico prediction of rodent oral toxicity. Nucleic Acids Res. 2014, 42, W53-W58.

(42) Ecological Structure Activity Relationships (ECOSAR) Predictive Model, v. 2.0; United States Environmental Protection Agency: Washington, DC, 2017.

(43) Lauber, K.; Wagner, G.; Gygax, A. Flora Helvetica - Illustrierte Flora der Schweiz, 5th ed.; Haupt Verlag: Bern, Switzerland, 2012.

(44) Rasmussen, L. H.; Hansen, H. C. B.; Lauren, D. Sorption, degradation and mobility of ptaquiloside, a carcinogenic Bracken 
(Pteridium sp.) constituent, in the soil environment. Chemosphere 2005, 58, 823-835.

(45) Jessing, K. K.; Cedergreen, N.; Jensen, J.; Hansen, H. C. B. Degradation and ecotoxicity of the biomedical drug artemisinin in soil. Environ. Toxicol. Chem. 2009, 28, 701-710.

(46) von Kiparski, G. R.; Lee, L. S.; Gillespie, A. R. Occurrence and fate of the phytotoxin juglone in alley soils under black walnut trees. $J$. Environ. Qual. 2007, 36, 709-717.

(47) NaToxAq: Natural Toxins and Drinking Water Quality - From Source to Tap. http://natoxaq.ku.dk/ (accessed Oct 20, 2017).

(48) Angiosperm Phylogeny Group. An update of the Angiosperm Phylogeny Group classification for the orders and families of flowering plants: APG IV. Bot. J. Linn. Soc. 2016, 181, 1-20.

(49) Ordinance on the Handling of Organisms in the Environment; Ordinance 814.911; Swiss Federal Council: Bern, Switzerland, 2008. Available at https://www.admin.ch/opc/en/classified-compilation/ 20062651/index.html.

(50) Commission Implementing Regulation (EU) 2016/1141 of 13 July 2016 adopting a list of invasive alien species of Union concern pursuant to Regulation (EU) No 1143/2014 of the European Parliament and of the Council; Official Journal of the European Union, 2016.

(51) Shinbo, Y.; Nakamura, Y.; Altaf-Ul-Amin, M.; Asahi, H.; Kurokawa, K.; Arita, M.; Saito, K.; Ohta, D.; Shibata, D.; Kanaya, S. KNApSAcK: A Comprehensive Species-Metabolite Relationship Database. In Plant Metabolomics; Saito, K., Dixon, R. A., Willmitzer, L., Eds.; Springer: Berlin, 2006; pp 165-181.

(52) Rasmussen, L. H.; Kroghsbo, S.; Frisvad, J. C.; Hansen, H. C. B. Occurrence of the carcinogenic bracken constituent ptaquiloside in fronds, topsoils and organic soil layers in Denmark. Chemosphere 2003, 51, 117-127.

(53) Hoerger, C. C.; Schenzel, J.; Strobel, B. W.; Bucheli, T. D. Analysis of selected phytotoxins and mycotoxins in environmental samples. Anal. Bioanal. Chem. 2009, 395, 1261-1289. 